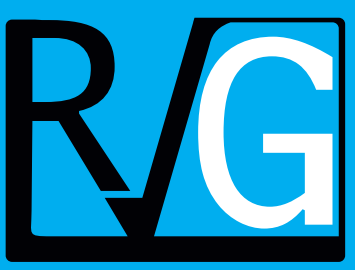

Año 21 No. 74

Abril - Junio 2016

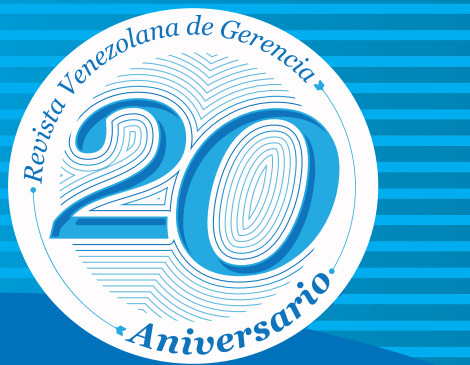

Venezolana de
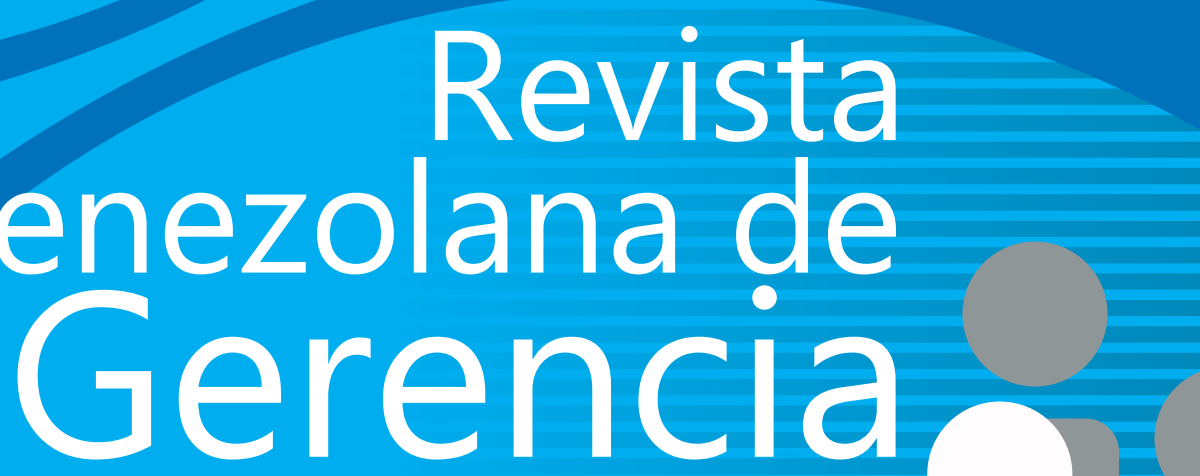


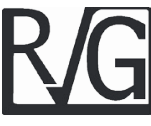

\title{
Innovación en instituciones de salud como fuente de competitividad
}

\author{
Gómez Calderón, Hernán ${ }^{1}$ \\ Londoño Rúa, John Edisson \\ Serrano Garcia, Jakeline
}

\begin{abstract}
Resumen
La estrategia Cluster le ha permitido a la ciudad de Medellín en Colombia, identificar sus capacidades productivas y enfocar el desarrollo de las mismas, ejemplo de ello es el plan de desarrollo 20082011 donde se estructura el plan de Ciencia, Tecnología e Innovación 2011-2021 como base para construir el futuro de ciudad líder, orientada a una alta competitividad apoyada en el desarrollo de la innovación. Por ende, este estudio pretende identificar el aprovechamiento de los recursos que brinda el entorno local, regional y global para desarrollar innovación, consultar los modelos de innovación existentes y determinar incidencias en la competitividad. Para ello se implementó una metodología de tipo cualitativa, a la vez que fueron realizadas entrevistas a profundidad en dos casos de estudio representados por instituciones innovadoras del Cluster de medicina y odontología. Se encontró que hay conciencia de la importancia de la innovación para desarrollar una mayor competitividad en los mercados que atienden las instituciones reflejado en que la innovación hace parte de sus objetivos estratégicos, desarrollando procesos en su implementación, con asignación de recursos y además con conciencia de la relevancia de interactuar con otros actores del mercado especialmente del cluster.
\end{abstract}

Palabras clave: innovación; cluster; competitividad; instituciones de salud.

Recibido: 10.09.15 Aceptado: 20.05.16

1 Docentes Instituto Tecnológico metropolitano ITM, hernangomez@itm.edu.co, johnlondono@ itm.edu.co, Jakelineserrano@itm.edu.co. 


\title{
Innovation in Health institutions as a source of Competitiveness
}

\begin{abstract}
The implementation of a Cluster strategy in the City of Medellin in Colombia, has enable to identify their productive capacities and focus on the development of them, the evidence of this is the 20082011 development plan of Medellin, where contains the plan for science, technology and innovation structure (CTI) 2011-2021 as the basis to build the future leading city-oriented high competitiveness supported in the development of innovation. The purpose of this study is to identify the use of resources provided by the local, regional and global environment to develop innovation, consult existing models of innovation and competitiveness determine incidents. This requires a qualitative methodology - which was implemented-, where depth interviews were conducted in two case studies represented by innovative institutions Cluster of Medicine and Dentistry. It was found that there is awareness of the importance of innovation to develop more competitiveness in the markets which serve the institutions reflected that innovation is part of its strategic objectives, developing processes in its implementation, with resource allocation and also consciousness of the importance of interacting with other market players especially the cluster.
\end{abstract}

Key words: innovation; cluster; competitiveness; health institutions.

\section{Introducción}

La realidad económica mundial está determinada por un nuevo orden en el cual existe una interdependencia creciente de las economías nacionales y regionales hacia su transformación en nudos de densas redes de intercambio de inversiones, información y mercancías, así como polos de desarrollo y gestión del conocimiento (Cámara de Comercio de Medellín, 2009).

El éxito industrial requiere empresas que sean capaces de crear competencias tecnológicas en productos, procesos y funciones buscando obtener ventajas competitivas, las cuales son creadas dinámicamente por empresarios y gobiernos, mediante un conjunto de estrategias y relaciones interinstitucionales que buscan optimizar la creación de valor (Solleiro y Castañón, 2005). El desarrollo de ventajas competitivas en el contexto global dependerá en gran medida por las políticas que desarrollen los países y gobiernos para fomentarlas (Johnson et al, 2006).

El estado colombiano lo ha comprendido y por eso cuenta con una Política Nacional de Competitividad la cual contempla cinco pilares, uno de ellos es el desarrollo de sectores o cluster de clase mundial (Departamento Nacional de Planeación, 2008). En la ciudad de Medellín ${ }^{2}$, desde el 2006 se viene trabajando la iniciativa cluster en servicios

2 Medellín es la segunda ciudad en importancia en Colombia y capital del departamento de Antioquia; según proyecciones del Departamento Administrativo Nacional de Estadística -DANE-, Medellín cuenta en 2015 con una población de 2.464 .322 habitantes, lo que la hace la segunda ciudad más poblada de Colombia. Económicamente es una ciudad que sobresale como uno de los principales centros financieros, industriales, comerciales y de servicios de Colombia, primordialmente en los sectores textil, confecciones, metalmecánico, eléctrico y electrónico, telecomunicaciones, automotriz, alimentos y salud (Medellín cómo vamos, 2015). 
de medicina y odontología ${ }^{3}$, basados en los logros que algunas instituciones locales ha tenido en el desarrollo de procesos vanguardistas a nivel mundial en servicios especializados de medicina (Marulanda et al, 2009).

Como parte del plan de desarrollo 2008-2011 de la Alcaldía de Medellín, se estructura el plan de Ciencia, Tecnología e Innovación (CTI) 2011-2021, como base para construir el futuro de ciudad líder, orientada a una alta competitividad apoyada en el desarrollo de la innovación (Pineda y Scheel, 2011). Dentro de los objetivos generales de desarrollo del plan CTI, se contemplan entre otros, promover proyectos de ciencia tecnología e innovación entre el sector académico y los sectores productivos y sociales, con énfasis en las cadenas productivas de salud, energía y tecnologías de la información y comunicación mediante el fomento de incorporar sistemas de gestión de la tecnología, la innovación y el conocimiento como funciones de la dirección estratégica organizacional moderna.

Lo anterior dio pie a identificar la innovación como una opción estratégica para incrementar la competitividad en empresas e instituciones del Cluster de Servicios de Medicina y Odontología de Medellín (CSMO), analizando la situación actual y perspectivas futuras para aprovechar las políticas de fortalecimiento y promoción de la innovación, propuestos en el plan de CTI de la ciudad de Medellín para ser más competitivos y lograr índices de sostenibilidad.

Para el desarrollo de la investigación, fue necesario entender como está conformado el CSMO de la ciudad de Medellín, el cual surge de la estrategia comunidad cluster liderada por la Alcaldía de Medellín, la Cámara de Comercio de Medellín para Antioquia, algunos empresarios de la región y el apoyo de diversas instituciones, que buscan promover el desarrollo y la competitividad empresarial (Cámara de Comercio de Medellín, 2013a).

El interés de la investigación, estuvo centrado en el CSMO, el cual propende por una cultura de confianza e integración institucional para la construcción de redes de cooperación, educación e investigación en salud, con el propósito de atraer al mercado internacional y contribuir al mejoramiento de la competitividad empresarial regional (Cámara de Comercio de Medellín, 2013).

El CSMO busca desarrollar actividades como: la internacionalización del cluster y sus servicios de salud; el desarrollo e implementación del modelo de atención a pacientes internacionales; la acreditación internacional de las instituciones prestadoras de servicios de salud - IPS; el fortalecimiento empresarial de la cadena productiva con énfasis en las empresas proveedoras de las IPS. Estas actividades se soportan en: innovación, talento humano, conformación de redes, prospectiva e investigación (Cámara de Comercio Medellín, 2014).

En la investigación se aplicaron entrevistas a profundidad, dirigidas a obtener por parte de los funcionarios de las instituciones del CSMO información que diera respuestas a cómo la innovación puede representar una opción estratégica que lleve a incrementar la competitividad en las mismas. La estructura de la entrevista a profundidad se basó en los objetivos de la investigación, que llevó a identificar unas categorías de información obtenidas en las respuestas y mediante

3 Por motivos de acuerdos de confidencialidad con las instituciones, se omite indicar su razón social y el nombre de la persona entrevistada. 
el aplicativo Atlas $\mathrm{Ti}^{4}$. De igual forma, permitió establecer relaciones existentes o no entre ellas, constituyéndose en la base de los resultados de la investigación.

Para el análisis y presentación de resultados, se tomó la información entregada por dos instituciones ${ }^{5}$ que hacen parte del consejo asesor del cluster y que actualmente están desarrollando en forma consciente procesos de innovación, enfocados a lograr una mayor competitividad en los mercados a los cuales ofrecen sus servicios, y cuya participación en el cluster son ejemplo de liderazgo y desarrollo de la innovación dados sus grandes inversiones y desarrollos en investigación y desarrollo.

\section{Innovación y competitividad en instituciones de salud}

En esta sección se parte de la revisión de literatura con la finalidad de establecer los conceptos de competitividad e innovación y la forma como la ciudad de Medellín las ha implementado en aras de fomentar la sostenibilidad en los mercados de las empresas e instituciones de salud a nivel regional, nacional e internacional.

\subsection{Competitividad y cluster}

La competitividad depende hoy día y en mayor medida en la generación de innovaciones y en la apropiación de recursos para el proceso de la misma. $\mathrm{Su}$ enfoque está en generar ventajas competitivas por parte de las empresas, con el propósito de tener una ventaja respecto a sus competidores (González et al, 2011).

Porter (2009) afirma que la competencia determina el éxito o fracaso de las empresas e igual expresa la conveniencia de las actividades que pueden favorecer su desempeño como innovaciones, una cultura cohesiva o una adecuada implementación. Habla de la estrategia competitiva como la búsqueda de una posición favorable dentro de una industria, escenario fundamental donde se lleva a cabo la competencia, cuya finalidad es establecer una posición rentable y sustentable frente a las fuerzas que rigen la competencia en la industria. Igual considera que las naciones logran el éxito competitivo en determinados sectores dadas las condiciones del entorno en que sea más progresivo, dinámico y estimulante (Porter, 2003).

En la teoría propuesta por el grupo de investigadores del Instituto Alemán del Desarrollo (Esser et al, 1996) se define la competitividad sustentada en la integración social, que propone a la vez reformas económicas junto con un proyecto de transformación de la sociedad, la que conceptualizan como competitividad sistémica. El concepto constituye un marco de referencia para los países tanto desarrollados como en vía de desarrollo. Establece dos elementos para determinar los factores de la competitividad industrial: la diferenciación entre cuatro niveles analíticos distintos (meta, macro, meso y micro) (Cuadro 1), y la vinculación de elementos pertenecientes a la economía industrial, a la teoría de la innovación y a la sociología industrial (Esser et al, 1996).

4 Es un software que permite codificar y analizar los datos de investigaciones cualitativas, ayudando a organizar, reagrupar y gestionar las categorías identificadas y la relación que existe entre ellas.

5 Por razones de confidencialidad en este artículo no se nombran las instituciones participantes en la entrevista a profundidad. 


\section{Cuadro 1 \\ Determinantes de la competitividad sistémica}

\begin{tabular}{|c|c|c|c|}
\hline Nivel micro & Nivel meso & Nivel macro & Nivel meta \\
\hline $\begin{array}{c}\text { Eficiencia, calidad, } \\
\text { flexibilidad en los } \\
\text { negocios }\end{array}$ & $\begin{array}{c}\text { Promoción } \\
\text { exportaciones }\end{array}$ & Política comercial & Cohesión social \\
\hline $\begin{array}{c}\text { Aprender } \\
\text { colectivamente }\end{array}$ & $\begin{array}{l}\text { Política de } \\
\text { localización }\end{array}$ & Política monetaria & $\begin{array}{c}\text { Capacidad de } \\
\text { formular } \\
\text { visiones y } \\
\text { estrategias }\end{array}$ \\
\hline $\begin{array}{c}\text { Estrategia de } \\
\text { negocios }\end{array}$ & Política regional & $\begin{array}{c}\text { Política } \\
\text { antimonopolio }\end{array}$ & $\begin{array}{c}\text { Nivel social del } \\
\text { empresario }\end{array}$ \\
\hline \multirow[t]{3}{*}{$\begin{array}{c}\text { Corporación formal } \\
\text { e informal }\end{array}$} & Política industrial & \multirow[t]{3}{*}{ Política fiscal } & $\begin{array}{c}\text { Organización } \\
\text { económica y } \\
\text { política } \\
\text { orientada al } \\
\text { desarrollo }\end{array}$ \\
\hline & Política tecnológica & & Memoria \\
\hline & Política ambiental & & colectiva \\
\hline
\end{tabular}

Fuente: Elaboración propia a partir de Gracia (2006).

La teoría de la competitividad sistémica presentada por Esser et al., (1996) reflexiona y argumenta que las exigencias de mercado internacional a las empresas nacionales cada vez son mayores, por lo que se ven obligadas a competir en forma de cluster industriales (Gracia, 2006). Por lo tanto la competitividad empresarial dependerá de que tanto el entorno apoye o no su mejoramiento, la eficiencia de las relaciones con las instituciones que las rodean, junto con las políticas macroeconómicas impulsadas por los países, más allá de sus recursos y capacidades internas.

Las empresas son instituciones que compiten en los mercados, y su mayor nivel de competitividad está en que sus capacidades se puedan reforzar si el entorno territorial en el que se desempeñan facilita el ambiente propicio para ello. La gestión pública puede inducir el desarrollo de redes de empresas organizadas con asiento en un particular territorio (cluster), donde la colaboración y la asociatividad de las empresas son elementos centrales para impulsar su competitividad.

Para Solvell (2008) el origen y surgimiento de los cluster está en la aglomeración natural de las empresas, que tiende a concentrarse en regiones determinadas, que permite la especialización regional y las actividades de los integrantes. De acuerdo a esto Hsu et al, (2013) lo admite como un grupo de entidades económicas que han establecido relaciones debido a la proximidad geográfica. 
Las empresas que deseen estar en la competición internacional necesitan pertenecer a redes interoganizacionales, que les permita acceder a recursos, a reducir información asimétrica entre empresas, aumentar su poder de negociación frente a los competidores y a la vez incrementar sus capacidades competitivas (Giuliani, 2013), en este sentido el cluster brinda la oportunidad de fomentar unas relaciones de crecimiento y agrupación industrial con las organizaciones que lo conforman posibilitando el acceso a los recursos estratégicos, al conocimiento colectivo, a las tecnologías, a la información y las infraestructuras (Hsu et al, 2013).

En síntesis, los cluster son agrupaciones industriales con ánimo de lucro, que buscan el bienestar económico general, que para lograr ser sostenibles y alcanzar la competitividad deben interactuar y complementar con los recursos y capacidades organizacionales de las empresas o instituciones que lo componen.

\subsection{Innovación}

Las empresas en los mercados internacionales para lograr mayores ventajas competitivas deben hacerlo a través de actos de innovación, por lo tanto deben buscar la forma de hacer cosas nuevas o mejorar lo que tienen para competir (Porter, 2003).

La Organización para la Cooperación y el Desarrollo Económicos -OCDE- (2005) define la innovación como la introducción de un nuevo, o significativamente mejorado, producto (bien o servicio), de un proceso, de un nuevo método de comercialización o de un nuevo método organizativo, en las prácticas internas de la empresa, la organización del lugar de trabajo o las relaciones exteriores. En tal sentido, los procesos de innovación de las organizaciones deben ofrecer una respuesta a la incertidumbre de los mercados como a las oportunidades existentes en la actualidad, dadas las circunstancias atribuibles a la globalización, por ejemplo el cambio en el comportamiento de consumo de los clientes, desarrollo de nuevas tecnologías en transporte y comunicaciones, mayor accesibilidad a nuevos mercados, facilidad de movilización de capitales y mano de obra entre otros, lo que representa el aumentar el potencial de nuevos clientes ubicados en cualquier parte del mundo. Por eso, si las empresas quieren ser exitosas y líderes dentro de un mercado tan competitivo como el actual, debe nutrirse constantemente de ideas, en este punto, la innovación aparece como una excelente alternativa.

La innovación puede ser analizada desde los diferentes procesos que llevan a cabo las organizaciones para sacar adelante sus proyectos de innovación. Sin embargo, en lo que respecta al estudio del proceso de la innovación no existe un modelo explicativo claro y definitivo (Velasco et al., 2007). Esta afirmación permite identificar que aunque existen varios modelos, ninguno reflejará la realidad de las empresas en el camino para alcanzar la innovación.

Chesbrough (2011) comentado por West et al, (2014) abrió un camino para cambiar la práctica de la innovación, el concepto de innovación abierta que propuso, llevó a las empresas ampliar sus fronteras en la creación y comercialización de innovaciones. La innovación abierta contempla que el conocimiento requerido para llegar a la innovación provenga de fuentes externas a la organización, no solamente del interior de las empresas. También considera la ampliación de los mercados para el uso externo de la innovación generada internamente que no sirva o pueda ser utilizada en el modelo actual de negocio de la empresa. 
Este paradigma asume que "las empresas pueden y deben utilizar ideas externas, así como las ideas internas y caminos internos y externos al mercado, ya que buscan avanzar en su tecnología" (Chesbrough et al., 2006: 1). Según Robledo (2013) la innovación abierta no pretende reemplazar otras formas de innovación, sino que propone la existencia de un amplio potencial de oportunidades de exploración y explotación del conocimiento tecnológico que residen por fuera de la organización.

Por ende el desarrollo de capacidades de articulación entre empresas, incluso en actividades de investigación conjunta y cooperación toma un papel más activo para la innovación. También es evidente la necesidad de desarrollar actividades de búsqueda de conocimiento externo a la organización para lo cual herramientas como la vigilancia tecnológica $\mathrm{o}$ la inteligencia competitiva resultan muy útiles para la implementación de procesos de innovación abierta, en donde la apuesta cluster genera el ambiente propicio para la innovación abierta.

Chesbrough et al, (2006) plantean que la innovación es una dimensión critica del desarrollo de la estrategia del negocio, para ejecutar y defender la ventaja competitiva. Los modelos de negocio utilizan tanto ideas internas como externas para crear valor, mientras define mecanismos internos para reclamar parte de ese valor (West et al, 2014). La innovación abierta asume que las ideas internas pueden ser llevadas al mercado a través de canales externos fuera del actual modelo de negocio para generar valor adicional. La premisa básica de la innovación abierta es abrir los procesos de innovación (Huizingh, 2010).

"Desde esta concepción de la innovación, la sociedad, en su conjunto, ha de estar abierta a los nuevos conocimientos, a las reglas y a los valores de la investigación y a la innovación con el convencimiento de que todas las instituciones $y$ organizaciones, en acción conjunta, pueden ser más innovadoras en los diferentes ámbitos (sociales, culturales, económicos, políticos, entre otros) y más competitivas de forma sostenible e integral" (Bernal et al, 2015: 265).

Para los negocios, la innovación es una manera más rentable para innovar, porque reduce costos, acelera la entrada al mercado, incrementa la diferenciación en el mercado y crea nuevas fuentes de ingresos para la organización (Chesbrough, 2011). El manejo de la innovación va más allá de un seguimiento a otras áreas, la tendencia en la subcontratación, la agilidad y la flexibilidad, han estimulado a las empresas a cambiar sus estrategias y procesos en otras áreas, inclusive en conformar redes de organizaciones (Huizingh, 2010).

A pesar de que la innovación abierta es popular entre los académicos y profesionales, en la gestión de la innovación aún se carece de una estructura que permita llevar a las empresas una implementación de su concepto en su totalidad, pues solo se hace en forma fragmentada (Nerone et al, 2014). Sin embargo, en la práctica su desarrollo ha sido importante puesto que los responsables en las empresas le ha reconocido el papel que juega la innovación abierta en el alcance de los objetivos estratégicos con desarrollo de la colaboración externa para generar y entregar innovación y el impacto que puede tener en la reducción de costos para usar y crear tecnología (West et al, 2014). 


\subsection{Competitividad e innovación en Medellín}

La corporación Ruta N, definida como "el centro de innovación y negocios de la Alcaldía de Medellín, es creada con el fin de potenciar nuevos negocios basados en el conocimiento, (...) a través del fomento, el desarrollo y el fortalecimiento del ecosistema de la ciencia, la tecnología y la innovación" (Pineda y Scheel, 2011). Como tarea al 2021, Ruta N, junto con la Alcaldía de Medellín, y a través de la ejecución del plan de Ciencia, Tecnología e Innovación (CTI), pretenden que la ciudad de Medellín llegue a ser una ciudad competitiva que crea riqueza por medio de acciones, tecnología e innovación, que genere empleos a través de actividades productivas de alto desempeño, desarrollando acciones concretas para dar prioridad a tres cadenas productivas: la cadena de la energía, la cadena de la salud y la cadena de las tecnologías de la información y comunicación.

En el caso de la cadena de salud, está justificado que el sector de la salud en Medellín ha logrado un desarrollo relevante durante un largo período de tiempo. Primero, alrededor de sus facultades de medicina, enfermería, odontología y salud pública; luego bajo el impulso de los diferentes programas tanto de investigación y desarrollo como de los exitosos grupos de trasplantes, y más recientemente de la mano de clínicas y hospitales que han logrado extender los servicios de sus instituciones a pacientes internacionales (Cámara Comercio de Medellín, 2013).

\section{Competitividad e innovación del Cluster de Servicios de Medicina y Odontología de Medellín: Resultados y consideraciones}

Con base en las ideas desarrolladas sobre competitividad e innovación y el proceso que ha llevado el CSMO de Medellín, se realizó un análisis basado en las dos instituciones estudiadas, que consistió en: identificar el aprovechamiento de los recursos que brinda el entorno local, regional y global para desarrollar innovación; en consultar los modelos de innovación existentes y determinar ¿cuáles y de qué forma los modelos se han apropiado en las innovaciones implementadas en las empresas e instituciones del CSMO?; a nivel de las empresas e instituciones reconocer ¿cuáles son las áreas, procesos, productos y servicios donde se evidencia algún tipo de generación de innovación?; y según los resultados ¿cuál ha sido el impacto logrado en materia de competitividad?

Para el análisis de los resultados se tomó como base las relaciones entre los códigos creados en Atlas $\mathrm{Ti}$ e identificados como propios de cada uno de los puntos de discusión, a través de redes semánticas. Éstas muestran las relaciones de significado entre los códigos, es decir representan la relación existente entre las diferentes variables cualitativas que se tomaron para el estudio. Esta relación se hace a partir de lo identificado en la revisión de la literatura y que pudo ser comprobado mediante los casos de estudio.

En resultados de innovación se presentan casos específicos de dos instituciones, los cuales por cuestiones de confidencialidad no son identificadas, sin embargo se pueden reconocer las principales áreas en las que se inscriben. Incluso para una de las instituciones esto les ha permitido ganar premios y reconocimientos de calidad y en las áreas específicas (ejemplos de áreas: Neonatos, gestión de ideas, prendas médicas, aparatos ortopédicos, prótesis, entre otras). 


\subsection{Determinación del uso de programas y mecanismos}

Para la determinación del uso de programas y mecanismos buscando promover el desarrollo de la innovación se preguntó a los entrevistados acerca de los principales tópicos en relación a este punto que se dan en la región y se indagó acerca de la posibilidad del uso de otros (diagrama 1).

\section{Diagrama 1}

\section{Red Semántica de Instituciones y Programas}

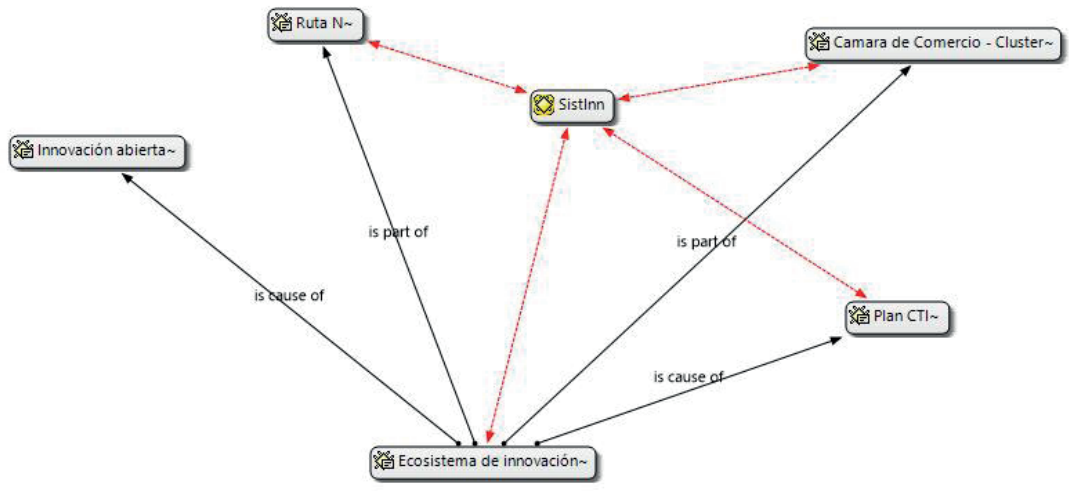

Fuente: Elaboración propia a partir de Atlas Ti

En el gráfico se muestra que el uso de los programas y mecanismos por parte de las dos instituciones en términos de innovación es común, se pudo evidenciar como la relación con estos apoyos es positiva y ha contribuido de algún modo a los desarrollos que se han tenido en temas de innovación, es decir, se han ejecutado trabajos mediante la colaboración de Ruta $\mathrm{N}$ y la Cámara de Comercio tanto en la definición como en la aplicación de procesos de innovación al interior de las instituciones.

Igualmente, se han apoyado en las convocatorias de Colciencias, quien es el ente Coordinador del Sistema Nacional de Ciencia, Tecnología e Innovación (Sistinn) para el desarrollo de sus proyectos de investigación. En el caso de la institución 1 , Ruta $\mathrm{N}$ ha apoyado en la capacitación de parte del equipo directivo en temas de gestión de la innovación, en la institución 2,
Ruta N los ha acompañado en el desarrollo de su modelo de negocio. En el caso del plan $\mathrm{CTI}$, la institución 2 plantea que es un plan ambicioso y desarrollado a muy a largo plazo en el cual se representa la posibilidad de mejoramiento de la visibilidad internacional y de compartir experiencias exitosas para el beneficio de la institución. En efecto se logra identificar que las instituciones hacen uso de los recursos que ofrece el ecosistema de innovación.

\subsection{Análisis del proceso de innovación en instituciones del clúster de servicios de medicina y odontología.}

Este análisis se basó en las actividades que las instituciones del Cluster realizan en materia de innovación, 
lo cual permitiría incluso determinar la aplicación de modelos específicos de Innovación (diagrama 2). Como tal un modelo de innovación estructurado no siguen las instituciones. No obstante, las instituciones destinan recursos, se capacitan y se está implementando una cultura del fomento y adaptación a la innovación. Particularmente, se puede identificar que la $I+D+i$ se realiza a partir de las necesidades propias de cada institución, las cuales se priorizan y se materializan en proyectos donde destinan recursos y talento humano para su desarrollo.

\section{Diagrama 2 \\ Red Semántica del Proceso de Innovación}

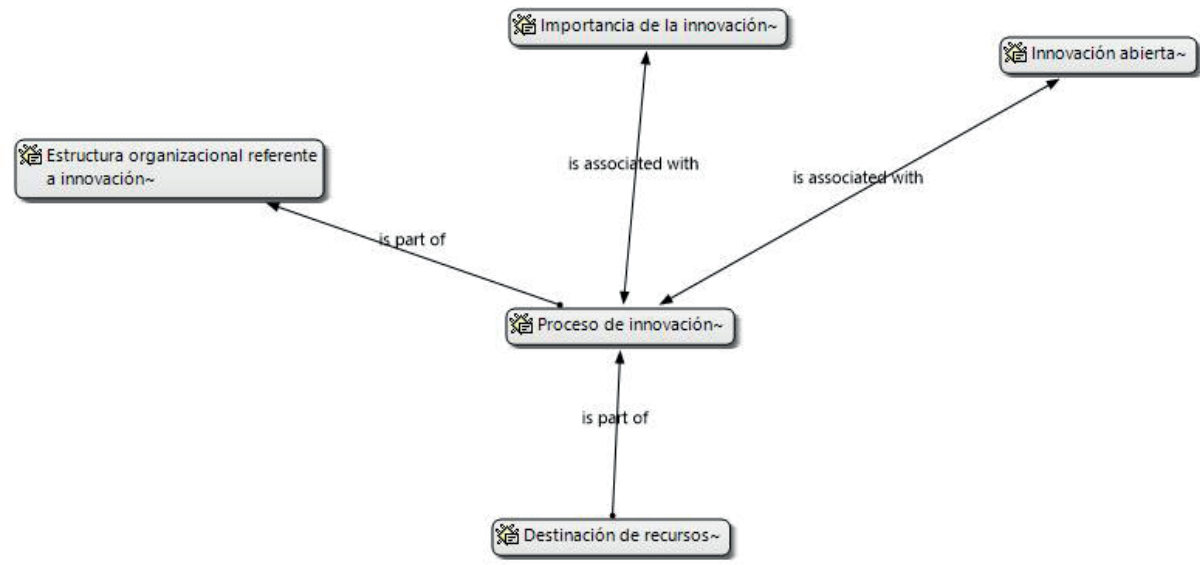

Fuente: Elaboración propia a partir de Atlas Ti

Se identifica que el proceso de innovación en ambas instituciones está en proceso de desarrollo e implementación, por lo que se ha avanzado solo en la definición de las actividades que hacen parte del mismo. En la Institución 1 se tiene establecido por política que la innovación es un tema estratégico y por ello se incorpora en la misión y visión de la organización.

Adicional a esto en esta institución los temas de innovación y en sí el sistema de gestión de la innovación se introduce como parte de la política integral de calidad. Para ello se definieron 4 procedimientos de gestión de la innovación, atendiendo la norma UNE 16002, es decir la NTC
5801. En la institución 2, es claro que una de las debilidades en innovación fue la centralización de la misma, lo cual no permitía desarrollar planes más concretos. La junta directiva de la institución les aprobó la creación de dirección científica con el fin de mantener el direccionamiento estratégico, pero la innovación como tal sigue en cabeza de cada área.

En el tema de innovación abierta, que tiene relación con un modelo determinado, se observa la aplicación de esta tendencia en ambas instituciones. En el caso de la Institución 2, se está buscando mediante el apoyo del Cluster la transferencia de conocimiento, por medio 
de pasantías con profesionales de toda América Latina y del Caribe.

La asignación de recursos para la innovación se hace con recursos propios destinados a la inversión, en ambas instituciones no se visualizan mecanismos de búsqueda de recursos frescos para la innovación, claro está sin tener en cuenta los recursos de conocimiento aportados en los programas y mecanismos de los que se hace uso. La estructura para la innovación aún es muy débil, aunque en ambas instituciones se encuentran fortalezas en la $I+D$ por la relación que ambas tienen con Universidades de la Región. También se observa que el Cluster viene adelantando procesos de vinculación con universidades para fortalecer la estructura académica de la transferencia de conocimiento.

\subsection{Establecimiento de áreas, procesos, productos, mercados y modelos de negocios con mayor grado de innovación en el cluster.}

La estructura definida para este punto está dada por la identificación de innovaciones en las áreas internas de la empresa, la gestión de la propiedad intelectual y los resultados específicos de innovación (diagrama 3)

\section{Diagrama 3 \\ Red Semántica de Resultados de Innovación}

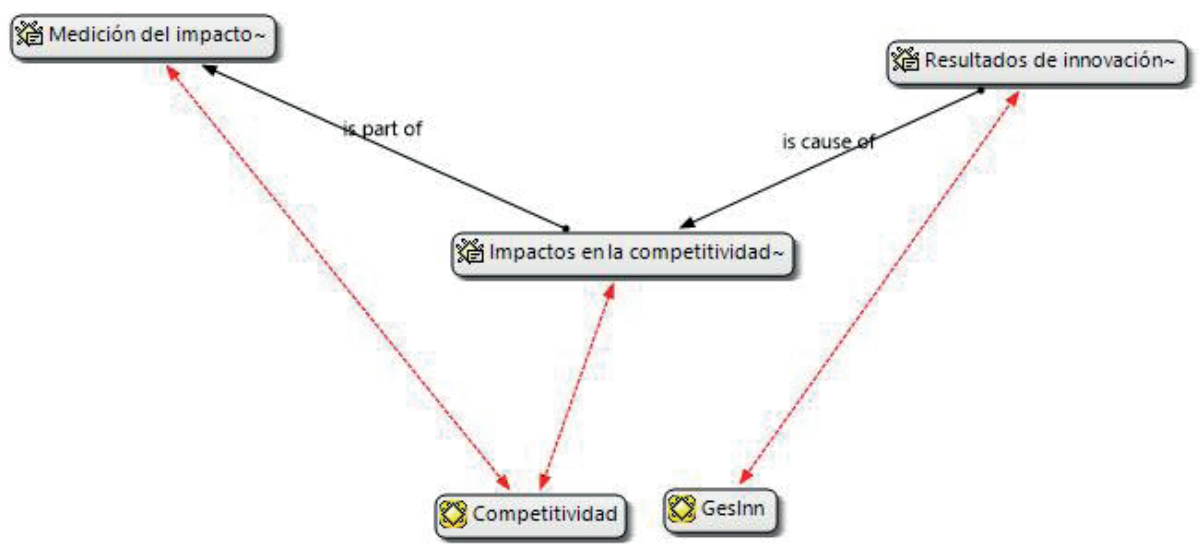

Fuente: Elaboración propia a partir de Atlas Ti

En el tema de gestión de la propiedad intelectual para la Institución 1, no se tiene una política de protección de la propiedad industrial, solo se cuenta con políticas de derechos de autor que están inmersas en la política de investigación como tal. La institución 2, muestra resultados en cuanto a patentes, pero se manifiesta que hace parte del resultado del proceso de investigación de la universidad en la cual se inscriben y no de la institución como tal. Adicional a esto, se presenta el problema de la comercialización de los resultados de las investigaciones, es decir 
en la transferencia tecnológica, donde la estructura de personal y el nivel de conocimiento del tema no son los más adecuados.

\subsection{Evaluación de los resultados de innovación en el desarrollo y competitividad del cluster}

La competitividad en el cluster está determinada por la propiedad intelectual representada en la creación y gestión de los desarrollos tecnológicos; los incentivos para el fomento y aplicación de la innovación, como lo es la autonomía para la investigación, la posibilidad de implementar los recursos en sus procesos médicos y las retribuciones económicas a la innovación. Por otra parte, se hace necesario resaltar que los impactos en la competitividad no se lograron identificar, debido a que las instituciones no cuentan con indicadores que permitan medir su alcance. Sin embargo, de manera informal, ambas instituciones manifiestan haber obtenido mejoramiento en el servicio y posibles impactos en la rentabilidad (diagrama 4).

\section{Diagrama 4 \\ Red Semántica de Competitividad}

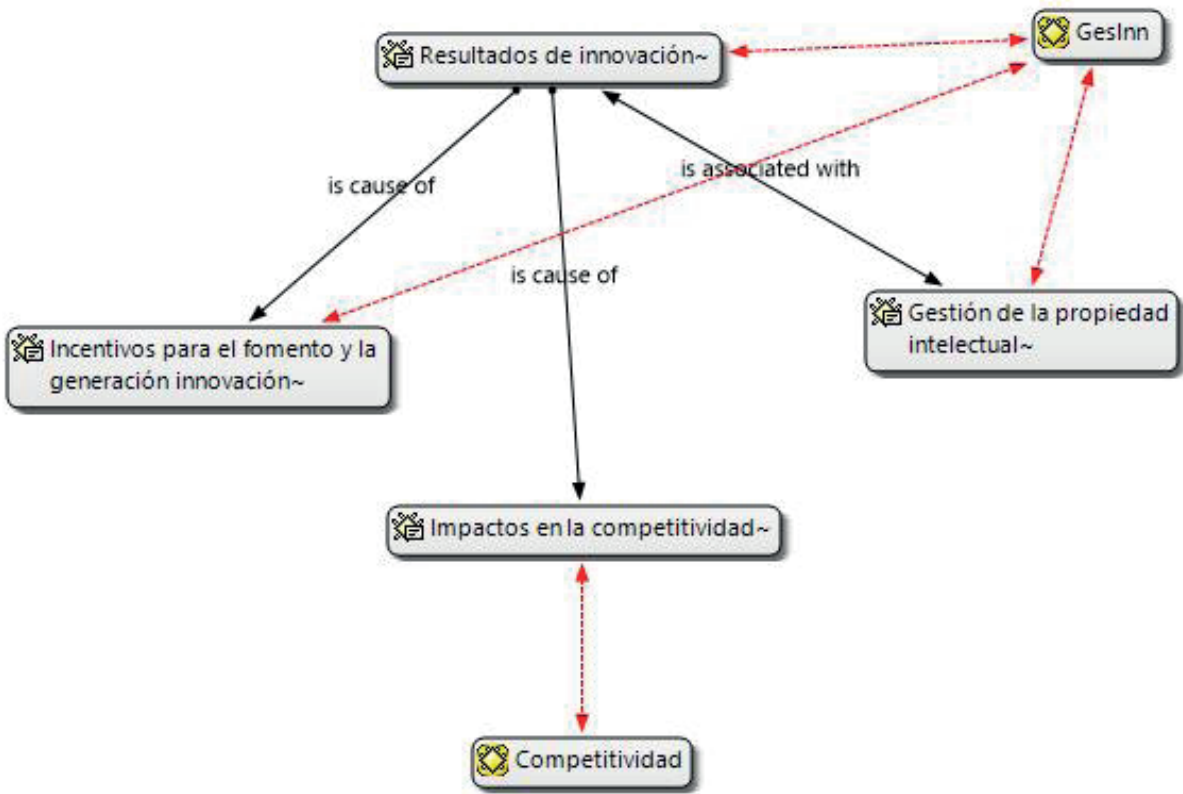

Fuente: Elaboración propia a partir de Atlas $\mathrm{Ti}$

Para la medición del impacto, estas instituciones por el tipo de servicios que prestan, se orientan a los rankings internacionales. Es el caso de la Institución 2 , donde se manifiesta que a pesar que los rankings no hacen una medición 
exacta, se ha avanzado y tienen uno de los puntajes más altos en gestión del conocimiento. Como medida interna del impacto esta institución ha definido dos tipos de indicadores, el de proceso y el de impacto como tal. En el de proceso se miden las publicaciones o los resultados que aún no permean la labor de la institución y de impacto cuando permite mejorar condiciones específicas relacionadas con el paciente.

\section{Conclusiones}

Se puede establecer que hay conciencia de la importancia de la innovación para desarrollar una mayor competitividad en los mercados que atienden las instituciones, reflejado en que la innovación hace parte de sus objetivos estratégicos, desarrollando procesos en su implementación, existencia de asignación de recursos y además son conscientes de la relevancia de interactuar con otros actores del mercado especialmente el CSMO.

Puede afirmarse en términos de la implementación de estrategias de competitividad sistémica, que en el CSMO de Medellín existe una integración de actores sociales para lograr los objetivos del nivel meta y desarrollar acciones a nivel meso, por cuanto se ve una participación activa de la Alcaldía de Medellín a través de los planes de desarrollo y el apoyo de Ruta N, articulados mediante el plan CTI 2021 como una estrategia de entorno regional. Todo esto enmarcado de las políticas de competitividad e innovación que Colombia ha implementado para permitir a las regiones y sus empresas ser más competitivas en sus mercados locales e igual trascender esa competitividad a los mercados internacionales.

En términos de la definición de innovación abierta en la que se plantea la necesidad de las organizaciones de aprovechar las oportunidades de entregar, compartir y apropiarse del conocimiento, el CSMO es una alternativa que tienen las instituciones que permite compartir y transferir conocimiento para el desarrollo de la innovación, a partir de la integración de las instituciones, los programas y el aprovechamiento de las mismas para el fomento de la innovación.

Los cluster como agrupaciones industriales que son, están en la búsqueda de un bienestar económico general para garantizar una mayor competitividad y sostenibilidad, se puede afirmar, que las instituciones de salud estudiadas lo entienden así, por lo tanto lideran como entidades representativas del cluster el desarrollo de la innovación en productos, servicios o procesos nuevos, generando estímulos internos, utilizando recursos que les permite el ecosistema de innovación. Aunque como ellas mismas lo manifiestan, no hay indicadores específicos que logren evidenciar el impacto en su competitividad, si lo han percibido en la reputación o imagen como instituciones a seguir, que a la larga es una muestra de un mayor desempeño en el sector que compiten.

Con respecto al desarrollo de procesos y modelos, la literatura muestra una evolución desde lo que es I+D hasta la innovación abierta, en las instituciones estudiadas no muestran que las actividades de innovación respondan a un modelo especifico, debido a que han actuado de forma emergente para dar respuesta a las exigencias de competitividad del sector, por lo que hasta ahora están en la etapa de implementación y desarrollo, a pesar de ello han obtenido resultados que les hace ver la importancia de la innovación como fuente de competitividad, por lo que son conscientes de establecer indicadores dentro de sus procesos de innovación para evaluar su desempeño competitivo.

Asimismo se logró determinar en ambas instituciones, que existe un área 
encargada de la gestión de la innovación, la cual tiene destinada recursos físicos, económicos y talento humano para su ejecución como parte de esa conciencia de generar innovación en aras de una mayor competitividad.

De acuerdo a la información obtenida, podría deducirse que el desarrollo y fortalecimiento de la innovación como proceso que soporte la competitividad de las instituciones de salud, aún está en sus primeras etapas de formulación e implementación, que no siguen un modelo específico, sino que actúan a situaciones emergentes que se propician según las necesidades de cada institución; y CSMO en igual manera hasta ahora viene facilitando condiciones para que cada una de ellas puedan compartir y fortalecer esos proceso.

\section{Referencias bibliográficas}

Bernal Torres, César Augusto; Frost González, Salomón (2015), Innovación abierta en empresas colombianas: reto a superar. Revista Venezolana de Gerencia. Pag. 252-267.

Cámara de Comercio de Medellín (2014), Cluster Servicios de Medicina y Odontología. Medellín. Recuperado en: http://www.camaramedellin.com. $\mathrm{co/site/Cluster-y-Competitividad/}$ Comunidad-Cluster/ClusterMedicina-y-Odontologia.aspx

Cámara de Comercio de Medellín para Antioquia (2013). Diseño e implementación de un modelo cluster para la atención de pacientes internacionales en la ciudad de Medellín: Expectativas y prospectivas. Disponible en: http:// www.camaramedellin.com.co/ site/Portals/0/Documentos/2013/ CLUSTER\%20No.\%208.pdf

Cámara de Comercio de Medellín (2013a), Clúster y Competitividad. Disponible en: http://www.camaramedellin.com. $\mathrm{co} /$ site/Cluster-y-Competitividad.aspx
Cámara de Comercio de Medellín (2009), Documento Comunidad Cluster No. 5 Avances de la estrategia cluster en Medellín y Antioquia, 1-284. Recuperado en: http:// www.camaramedellin.com.co/site/ DesktopModules/Bring2mind/DMX/ Download.aspx?Command=Core Download\&Entryld $=200$ \&Portalld $=0$ \& Tabld $=515$

Chesbrough, Henry (2011), Everything you need to know about open innovation. Revista Forbes. Recuperado de http://www.forbes.com/sites/ henrychesbrough/2011/03/21/ everything-you-need-to-know-aboutopen-innovation/

Chesbrough, Henry, Vanhaverbeke, Wim., y West, Joel (2006), Open Innovation: Researching a New Paradigm. London: Oxford University Press.

Departamento Nacional de Planeación DNP (2008), Documento Conpes 3527. Política Nacional de Competitividad y Productividad. Bogotá D.C., 23 de junio de 2008.

Esser, Klaus; Hillebrand, Wolfgang; Messner, Dirk; \& Meyer-Stamer, Jörg (1996). Revista de la Cepal. Recuperado el 22 de septiembre de 2013, de http:// www.meyer-stamer.de/1996/cepal. htm

Giuliani, Elisa (2013). Networks dynamics in regional cluster: Evidence from Chile. Recuperado el dia 11 de junio de 2016 de http://itm.elogim. com:2112/S0048733313000796/1s2.0-S0048733313000796-main. pdf? tid=710bad9a-2fc9-11e6-87cb00000aab0f27\&acdnat $=1465645471$ a9d32afd4c9d0424c18f1a68e20b22 $\overline{0 b}$

González, Armando; Mendoza, Maria; y Juárez Senén. (2011). La innovación en el contexto competitivo de las empresas e instituciones. VI Congreso internacional de Sistemas de Innovación para la Competitividad.

Gracia, Maximiliano (2006). La competitividad sistémica; elemento de desarrollo regional y local. Ciencia y Mar, pág. 39-46.

Huizingh, Eelko. (2010). Open innovation: State of the art and future 
perspectives. Recuperado el día 25 de noviembre de 2014 de: http://www. sciencedirect.com/science/article/pii/ S0166497210001100

Johnson Gerry, Scholes Kevan y Whittington Richard. (2006). Dirección Estratégica. Pearson Prentice Hall, México, 7a edición.

Marulanda, Jorge; Correa, Geovanny y Mejía, Luis. (2009), El cluster de salud en Medellín, Ventaja competitiva alternativa para la ciudad. Revista EAN 67. Septiembre-Diciembre 2009.P.37-58 recuperado de: http:// journal.ean.edu.co/index.php/ Revista/article/view/483/471

Maw-Shin Hsu, Yung-Lung Lai y Feng-Jyh Lin. (2013). Effects of Industry Clusters on Company Competitiveness: Special Economic Zones in Taiwan. Department of Business Administration, Feng Chia University, Taichung 40724, Taiwan.

Medellín cómo vamos (2015). La ciudad. Recuperado el 9 de junio de 2016 de http://www.medellincomovamos.org/ la-ciudad.

Nerone, Murilo; Canciglieri Junior, Osiris y Liao, Yongxin. (2014). Clasificaction of the open innovation practices: the creativity level. Recuperado de http://www. researchgate.net/profile/Yongxin Liao2/publication/267863666_ Classification_of_the_Open_Innovation Practices the Creativity_LevelT links $/ \overline{5} 45 \mathrm{~b} 5 \mathrm{~b} 6 \mathrm{e} 0 \mathrm{cf} 2877 \overline{9} \mathrm{a} 4 \mathrm{dba} 8 \mathrm{ab}$. pdf

Organización para la Cooperación y el Desarrollo Económicos -OCDE(2005). Oslo Manual: Proposed guidelines for collecting and interpreting innovation data. European Commission y Eurostat, Third edition, Paris, 1-92
Pineda, Leonardo; Scheel, Carlos. (2011), Plan de Ciencia, Tecnología e innovación de Medellín 2011-2021. Universidad del Rosario en Alianza con la escuela de graduados en administración de empresas del Instituto de Monterrey México. Recuperado de: http://www. grupo-epm.com/Portals/1/biblioteca epm_virtual/Plan-de-CTi-de-Medellin. pdf

Porter, Michael. (2009). Ventaja competitiva. Creación y sostenimiento de un desempeño superior. Grupo Editorial Patria, México, $2^{\mathrm{a}}$ edición.

Porter, Michael. (2003). Ser Competitivos. Barcelona: Deusto

Robledo, Jorge. (2013). Introducción a la Gestión Tecnológica. Universidad Nacional de Colombia - Sede Medellín, Facultad de Minas.

Solvell, Örjan (2008). Clusters balancing evolutionary and constructive forces. 1a ed. Stockholm, Sweden: Ivory Tower Publishing.

Solleiro, José Luis y Castañón, Rosario. (2005). Competitiveness and innovation systems: the challenges for Mexico's insertion in the global context. Technovation 25, pág. 10591070

Velasco, Eva; Zamanillo, Ibon y Intxaurburu Gurutze (2007), Evolución de los modelos sobre el proceso de innovación desde el modelo lineal hasta los sistemas de innovación. $\mathbf{X X}$ Congreso anual de AEDEM, 2

West, Joel; Salter, Ammon, Vanharverbeke, Win y Chesbrough, Henry (2014), Open innovation: The next decade. Recuperado el 25 de noviembre de 2014 de http://ac.els-cdn.com/ S $0048733314000407 / 1$-s2.0 S0048733314000407-main.pdf? tid $=f 178819 a-143 d-11 e 5-a 31 d-$ 00000aacb35d\&acdnat $=1434469378$ d6c76bc72d68918316e2b255a5cca1 $6 e$ 


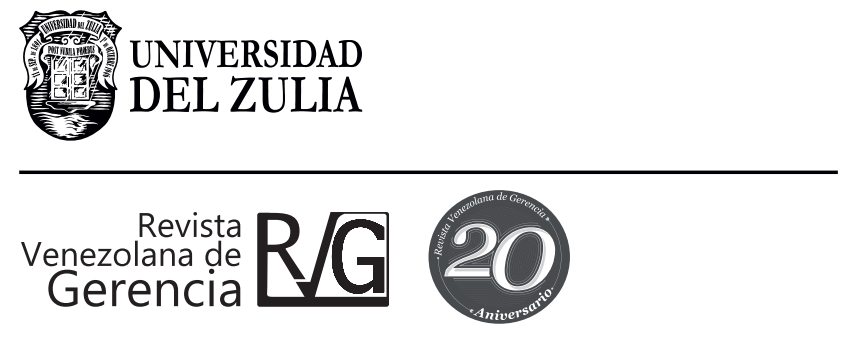

Año 21, No. 74

Esta revista fue editada en formato digital y publicada en junio del 2016, por la Universidad del Zulia, Vicerrectorado Académico, Serbiluz - Fondo editorial, Maracaibo -Venezuela. 